\title{
The Microwave Spectrum of Fluoroacetylene in Ground and Vibrationally Excited States and Some Laser Enhancement Effects
}

\author{
Harold Jones and H. D. Rudolph \\ Abt. Phys. Chemie, Universität Ulm, 7900 Ulm, West Germany
}

Z. Naturforsch. 34a, 340-352 (1979); received December 23, 1978

\begin{abstract}
The microwave spectrum of HCCF and DCCF has been investigated in all vibrational states with energy up to $1500 \mathrm{~cm}^{-1}$. In the ground state and low-lying vibrational states mm-wave measurements up to $210 \mathrm{GHz}$ were made. In some cases a detailed analysis of the vibrational state rotation spectrum including the effects of $l$-type resonance, and the determination of the anharmonicity constant $g_{\mathrm{tt}}$ was possible. The rotational spectra of combination states of the type $\left(v_{\mathrm{t}}=2, v_{\mathrm{t}^{\prime}}=1\right)$ were observed and partially analyzed, which is, to our knowledge, the first time this has been accomplished. The low-lying vibrational states of ${ }^{13} \mathrm{C}$ species of HCCF and DCCF were also observed.

The $9.4 \mu \mathrm{m} \mathrm{P}(14) \mathrm{CO}_{2}$-laser line was observed to produce a reduction in intensity in the ground state and an increase in intensity in the $v_{3}=1$ excited state $J=0 \rightarrow 1,1 \rightarrow 2,2 \rightarrow 3$ transitions of DCCF.
\end{abstract}

\section{Introduction}

Fluoroacetylene is one of the very small group of linear molecules containing four atoms which since the observation of non-linearity effects in HCNO (e.g. [1]) have aquired new interest. The haloacetylenes do not display such non-linearity effects, but the analysis of their rotational spectra, particularly in excited vibrational states, is of importance since it gives data on the behaviour of "well-behaved" linear molecules. A second reason why fluoroacetylene is of interest is that it possesses a strong infrared absorption band in the $10 \mu \mathrm{m}$ region, the $\nu_{3}$ (C-F stretch) band, which makes it a suitable candidate for experiments involving $\mathrm{CO}_{2}$ lasers. This latter property was in fact the main reason for our undertaking the relatively complex synthetic route necessary for the production of fluoroacetylene. The suitability of this molecule for such experiments has been demonstrated by Tanaka et al. [2] in their Stark laser-spectroscopy study. In laser-microwave double resonance experiments on deuterofluoroacetylene, we observed that the $9.4 \mu \mathrm{m} \mathrm{P}(14) \mathrm{CO}_{2}$ laser line caused a decrease in absorption in the ground state and an increase in absorption in the $\left(1,0^{0}, 0^{0}\right)$ state $J=0 \rightarrow 1$, $1 \rightarrow 2$ and $2 \rightarrow 3$ rotational transitions. (In this paper, since rotational transitions of no states involving the vibrational modes $\nu_{1}$ and $\nu_{2}$ were observed, we will designate the states involved by three quantum numbers only $\left(v_{3}, v_{4}, v_{5}\right)$. From a

Reprint requests to Dr. Harold Jones. Please order a reprint rather than making your own copy.

$0340-4811 / 79 / 0300-0340 \$ 01.00 / 0$ conventional infrared study of the $v_{3}$ band of DCCF it appeared that the most likely mechanism for the production of this effect was that this laser line pumped the $R(9)$ transition of this band, which to within the relative poor accuracy of conventional infrared measurements appeared to be coincident. In order to investigate this effect it was necessary to be able to make measurements in the region of $170 \mathrm{GHz}$. Since we were already involved in a considerable experimental investment in this molecule we decided to measure and analyze as much as possible of the excited state rotational spectrum of fluoroacetylene. We were able to measure the rotational spectrum of all vibrational states up to about $1500 \mathrm{~cm}^{-1}$ for both HCCF and DCCF and also the low-lying bending modes of the four ${ }^{13} \mathrm{C}$-species. The energy of the vibrational states in which rotational transitions were measured is shown schematically in Fig. 1 based on the infrared data of Hunt and Wilson [3]. The microwave spectrum of the ground state and the lowlying bending modes have been previously measured by Tyler and Sheridan [4]. We have been able to improve the accuracy of these measurements and in the case of the first excited state of the bending modes were able to observe direct $l$-type doubling transitions. The measurements carried out in the states $\left(0,(2,1)^{1}\right)$ and $\left(0,(1,2)^{1}\right)$ are to our knowledge the first carried out in such combination states.

The rotational spectrum of excited vibrational states of chloro- [5] and bromoacetylene [6], and in the case of fluoroacetylene [7] the transition frequencies of some of the excited states discussed here have been recently reported. 


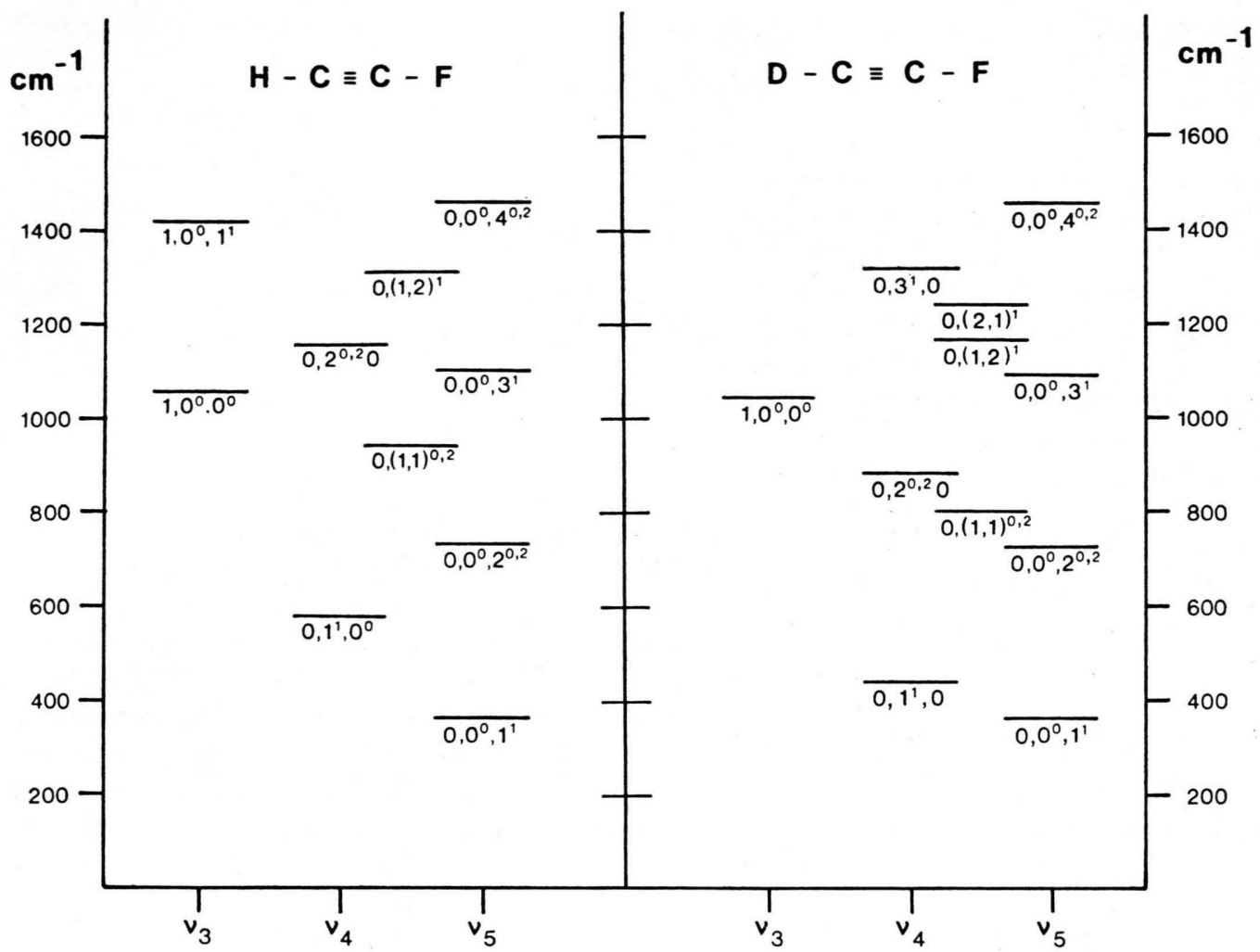

Fig. 1. Diagrammatical representation of the vibrational states of HCCF and DCCF investigated in this work. Only three quantum numbers $v_{3}, v_{4}, v_{5}$ are given and the indices indicate the values of $l$ in the state concerned.

\section{Experimental}

The sample of fluoroacetylene was produced by the method of Viche and Franchimont [8], from tribromethylene over pentabromoethane and 1-fluoro-1.1.2.2.tetrabromoethane. The resulting gaseous fluoroacetylene was collected over water and transferred into a glass bulb. Further purification, such as trap to trap distillation, was not carried out because of the hazards attached to such a procedure. Although gaseous fluoroacetylene appears to be harmless even when mixed with air, in the condensed phase the substance is capable of spontaneous explosion. A demonstration of this property was experienced. The sample used was known to contain water vapour and a little acetylene as impurities. Deuterated samples were produced by direct exchange with slightly alkaline $\mathrm{D}_{2} \mathrm{O}$.

Measurements up to $63 \mathrm{GHz}$ were made in a conventional $2 \mathrm{~m}$ long X-band Stark cell at room temperature. The mm-wave measurements were made using a cell consisting of a 1 metre length of
$\mathrm{RG}(98) / \mathrm{u}$ waveguide with a run-in harmonic generator and detector from Custom Microwave. Fundamental microwave power was supplied by Oki $50 \mathrm{~V} 11$ and $60 \mathrm{~V} 12$ klystrons. Source modulation at a frequency of $5 \mathrm{kHz}$ was employed.

The laser induced changes in microwave absorption were observed by introduction of 4 Watts $\mathrm{cw}$ $\mathrm{CO}_{2}$-laser power chopped at a frequency of $1 \mathrm{kHz}$ into the waveguide cells, followed by phasesensitive detection of the microwave signal.

The device used to couple in the infrared radiation into the cells without significant deterioration of the microwave transmission has been described previously [9].

\section{Theory}

A brief summary will be given of the expressions given by Winnewisser and Winnewisser [1] based on the formalism of Amat and Nielsen [10], for the energy levels and rotation transition frequencies of a linear molecule in the vibrationally excited states 
involving the bending modes. Expressions developed for the states $\left(0,(2,1)^{1}\right),\left(0,(1,2)^{1}\right)$ and $\left(0,0,4^{0,2}\right)$ which have not been previously treated in detail will also be given.

With the present data on fluoroacetylene terms in the constant $\varrho$ which arise from off-diagonal matrix elements of the form $\left\langle v_{\mathrm{t}}, l_{\mathrm{t}}\left|H_{2}\right| v_{\mathrm{t}}, l_{\mathrm{t}} \pm 4\right\rangle$ were found to be unimportant for all states except $\left(0,(1,1)^{0,2}\right)$ and these terms will be omitted from the following considerations. As discussed earlier [1] since $l$ is not a rigorous quantum number, the normal classification of states according to $l$ is not strictly correct and only a classification into symmetric and antisymmetric species is really meaningful. However since the observed transitions are most easily identifiable by virtue of their Stark effects as possessing a particular $l$-value the two methods of classification will be used.

\section{Second Excited State of a Degenerate Bending Mode, $V_{\mathrm{t}}=\mathbf{2}$}

In such a state we expect a nondegenerate level with $|l|=0$ and a doubly degenerate level with $|l|=2$. The degeneracy of the $|l|=2$ levels is raised by an interaction between the $|l|=0$ level and one of the $|l|=2$ levels via off-diagonal matrix elements $\left\langle v_{\mathrm{t}}, 0\left|H_{2}\right| v_{\mathrm{t}}, \pm 2\right\rangle$. Neglecting other offdiagonal elements we arrive at the frequency expressions.

$$
\begin{aligned}
v_{\mathrm{a}}= & 2(J+1) B_{\mathrm{v}}-4(J+1)^{3} D_{\mathrm{v}} \\
& +2(J+1) \Delta_{2}{ }^{1}, \\
v_{\mathrm{s}}{ }^{ \pm}= & 2(J+1) B_{\mathrm{v}}-4(J+1)^{3} D_{\mathrm{v}}+2(J+1) \Delta_{2}^{1} / 2 \\
& \pm\left\{1 / 2\left[\left(\Delta_{2}\right)^{2}+4 q_{\mathrm{t}}{ }^{2} f(f-2)\right]_{(J+1)}^{1 / 2}\right. \\
& \left.-1 / 2\left[\left(\Delta_{2}\right)^{2}+4 q_{\mathrm{t}}{ }^{2} f(f-2)\right]_{J}^{1 / 2}\right\}
\end{aligned}
$$

where $f=J(J+1)$ and

$$
\begin{aligned}
\Delta_{2} & =\Delta_{2}{ }^{0}+\Delta_{2}{ }^{1} J(J+1) \\
& +4\left(g_{\mathrm{tt}}-4 D_{\mathrm{v}}-B_{\mathrm{v}}-4 \gamma_{11}\right)+4\left[\gamma_{11}+2 D_{\mathrm{v}}\right] f .
\end{aligned}
$$

$g_{\mathrm{tt}}$ is the anharmonicity constant which describes the contribution of the vibrational angular momentum to the vibrational energy. $\gamma_{11}=$ rovibration interaction constant.

Thus as may be seen the frequency of the $v_{\mathrm{s}}{ }^{ \pm}$transitions is slightly dependent on $g_{\mathrm{tt}}$ and consequently it is possible to obtain an approximate value for this constant. The + and - signs of $v_{\mathrm{s}}{ }^{ \pm}$ refer simply to whether the positive or negative root was selected.
From the above expressions the following sum rules may be derived.

$$
\begin{aligned}
\left(v_{\mathrm{s}}++v_{\mathrm{s}}^{-}+v_{\mathrm{a}}\right)^{*}= & 3 B_{\mathrm{v}}+8 \gamma_{11}+16 D_{\mathrm{v}} \\
& -6(J+1)^{2} D_{\mathrm{v}} \\
\left(v_{\mathrm{s}}{ }^{+}+v_{\mathrm{s}}^{-}\right)^{*}= & 2 B_{\mathrm{v}}+4 \gamma_{11}+8 D_{\mathrm{v}} \\
& -4(J+1)^{2} D_{\mathrm{v}}
\end{aligned}
$$

where $v^{*}$ refers to $v / 2(J+1)$. Using Eqs. (1), (3) and (4) the constants $B_{\mathrm{v}}, \gamma_{11}$ and $D_{\mathrm{v}}$ may be calculated.

\section{State $v_{\mathrm{t}}=\mathbf{4}$}

In principle in such a state, sub-states $|l|=0$, 2 and 4 are possible, but in the present case since only transitions with $J \leqq 2$ were measured, $|l|=4$ transitions were not observed. Consequently the situation is very similar to the $v_{\mathrm{t}}=2$ states except that the off-diagonal element $\left\langle v_{\mathrm{t}}, 0\left|H_{2}\right| v_{\mathrm{t}}, \pm 2\right\rangle$ is now $\left\langle 4,0\left|H_{2}\right| 4, \pm 2\right\rangle$ and in this case has a value $\sqrt{3 / 2} q_{t}[f(f-2)]^{1 / 2}$ and this results in the term in Eq. (2) in $q_{\mathrm{t}}^{2}$ being multipled by a factor of three, i.e. the last term in the two square brackets of Eq. (2) become $12 q_{\mathrm{t}}^{2} f(f-2)$. The other equations remain unchanged.

States $v_{\mathrm{t}}=1$ or $v_{\mathrm{t}}=3$

Since transitions with only $J \leqq 3$ were measured only transitions identifiable with $|l|=1$ were observed in both $v_{\mathrm{t}}=1$ and $v_{\mathrm{t}}=3$ states. Consequently the well-known expression

$v^{*}=\left[B_{\text {eff }} \pm q_{\mathrm{t} / 4}(v+1)\right]-2 D_{\mathrm{v}}\left[(J+1)^{2}-l^{2}\right]$

is sufficient for our purposes. It should be noted that $B_{\text {eff }}=B_{\mathrm{v}}+\gamma_{11}+2 D_{\mathrm{v}}$.

For the $v_{\mathrm{t}}=1$ states direct $l$-type doubling transitions were measured and the transition frequencies were fitted to the expression

$$
v_{\mathrm{t}}=q_{\mathrm{t}}{ }^{0} J(J+1)-q_{\mathrm{t}}{ }^{1} J^{2}(J+1)^{2} .
$$

\section{Combination States}

$v_{\mathrm{t}}=1, v_{\mathrm{t}^{\prime}}=1$

In such a state $l_{\mathrm{t}}$ and $l_{\mathrm{t}^{\prime}}$ may combine to form two pairs of states with $l=0$ or $l= \pm 2$. The degeneracy of the two $l=0$ states is lifted by vibration $l$-type doubling [10], with the off-diagonal element responsible for this

$$
\begin{aligned}
& \left\langle v_{\mathrm{t}}, l_{\mathrm{t}} ; v_{\mathrm{t}^{\prime}}, l_{\mathrm{t}^{\prime}}\left|H_{2}\right| v_{\mathrm{t}}, l_{\mathrm{t}} \pm 2 ; v_{\mathrm{t}^{\prime}}, l_{\mathrm{t}^{\prime}} \pm 2\right\rangle \\
& \quad=\left\langle 1,-1 ; 1,1\left|H_{2}\right| 1,1 ; 1-1\right\rangle=r_{\mathrm{tt}^{\prime}} \\
& \text { where } r_{\mathrm{tt}^{\prime}}=r_{\mathrm{tt}^{\prime}}^{0}+r_{\mathrm{tt}^{\prime}}^{1} J(J+1) .
\end{aligned}
$$


The degeneracy of the $l= \pm 2$ states is raised by rotational $l$-type resonance.

In the foregoing considerations off-diagonal elements of the form $\left\langle v_{\mathrm{t}}, l_{\mathrm{t}}\left|H_{2}\right| v_{\mathrm{t}}, l_{\mathrm{t}} \pm 4\right\rangle$ have been neglected, they were observed to produce negligible contribution to the transition frequencies. However in this state such elements, specifically $\left\langle v_{\mathrm{t}}, l_{\mathrm{t}}, v_{\mathrm{t}^{\prime}}, l_{\mathrm{t}^{\prime}}-2\left|H_{2}\right| v_{\mathrm{t}}, l_{\mathrm{t}}, v_{\mathrm{t}^{\prime}}+2\right\rangle$, were observed to produce detectable effects and these give rise to terms in the constant $\varrho_{t t}$.

The following sum rules have been given [1] for this state.

$$
\begin{aligned}
& \left(v_{\mathrm{s}}{ }^{+}+v_{\mathrm{s}}{ }^{-}+\nu_{\mathrm{a}}{ }^{+}-v_{\mathrm{a}}^{-}\right)^{*} \\
& =4 B_{\mathrm{v}}+16 D_{\mathrm{v}}-8 D_{\mathrm{v}}(\mathrm{J}+1)^{2}, \\
& {\left[\left(v_{\mathrm{s}}^{+}+\boldsymbol{v}_{\mathrm{s}}^{-}\right)-\left(v_{\mathrm{a}}{ }^{+}+v_{\mathrm{a}}^{-}\right)\right]^{*}} \\
& =2\left(r_{\mathrm{tt}^{\prime}}^{1}-2 \varrho_{\mathrm{tt} \mathrm{t}^{\prime}}\right)+4(J+1)^{2} \varrho_{\mathrm{tt}}{ }^{\prime}, \\
& {\left[\left(v_{\mathrm{s}}{ }^{+}+v_{\mathrm{a}^{+}}{ }^{+}-\left(\nu_{\mathrm{s}}^{-}+v_{\mathrm{a}}^{-}\right)\right]^{*}\right.} \\
& \cong 4 \gamma_{11^{\prime}}+16 D_{\mathrm{v}} \text {. }
\end{aligned}
$$

Using these rules $B_{\mathrm{v}}, r_{\mathrm{tt}^{\prime}}^{1}$ and $\gamma_{\mathrm{ll}^{\prime}}$ can be determined.

$v_{\mathrm{t}}=2, v_{\mathrm{t}^{\prime}}=1$

It appears that the rotational spectrum of a linear molecule in this type of combination state has never been studied in detail. The $l$-value of the two states may be combined to produce 4 substates with $|l|=1$ and two with $|l|=3$, and the overall condition may be described by a $6 \times 6$ matrix. If

\begin{tabular}{|c|c|c|c|}
\hline & $2^{ \pm 2}, 1^{ \pm 1}$ & $2^{ \pm 2}, 1^{ \pm 1}$ & $2^{0}, 1^{ \pm 1}$ \\
\hline $2^{ \pm 2}, 1^{ \pm 1}$ & $E_{3}$ & $b^{\prime}$ & $a^{\prime}$ \\
\hline $2^{ \pm 2}, 1^{ \pm 1}$ & $b^{\prime}$ & $E_{2}$ & $R+a$ \\
\hline $2^{0}, 1^{ \pm 1}$ & $a^{\prime}$ & $R+a$ & $E_{1}+b$ \\
\hline
\end{tabular}
all off-diagonal elements in which the change in $\left|l_{\mathrm{t}}+l_{\mathrm{t}^{\prime}}\right|$ is greater than two are neglected (as in Ref. [10]), forming the symmetric and antisymmetric combinations results in two $3 \times 3$ matrices, the symmetric one of which is

The elements $a, a^{\prime}$ and $b, b^{\prime}$ can be derived from the general formula given by Nielsen and Shaffer [11] and $R$ is given by Amat and Nielsen [10] as

$$
\begin{aligned}
& a=(\sqrt{2})^{-1} q_{\mathrm{t}} J(J+1), \\
& a^{\prime}=(\sqrt{2})^{-1} q_{\mathrm{t}}\{(J(J+1)-6)(J(J+1)-2)\}^{1 / 2}, \\
& b=0.5 q_{\mathrm{t}^{\prime}} J(J+1), \\
& b^{\prime}=0.5 q_{\mathrm{t}^{\prime}}\{(J(J+1)-6)(J(J+1)-2)\}^{1 / 2}, \\
& R=\sqrt{2} r_{\mathrm{tt}^{\prime}}=\sqrt{2}\left(r_{\mathrm{tt}^{\prime}}^{0}+r_{\mathrm{tt}^{\prime}}^{1} J(J+1)\right)
\end{aligned}
$$

$$
\begin{aligned}
E_{1}=E_{\mathrm{v}}+B_{\mathrm{v}}(J(J+1)-1)-D_{\mathrm{v}}(J(J+1)-1)^{2} \\
E_{2}=E_{\mathrm{v}}+4 g_{\mathrm{tt}^{\prime}}-2 g_{\mathrm{tt}^{\prime}}+\left(B_{\mathrm{v}}-2 \gamma_{11^{\prime}}\right) \\
\cdot(J(J+1)-1)-D_{\mathrm{v}}(J(J+1)-1)^{2} \\
E_{3}=E_{\mathrm{v}}+4 g_{\mathrm{tt}}+2 g_{\mathrm{tt}^{\prime}}+\left(B_{\mathrm{v}}+2 \gamma_{\mathrm{Il}^{\prime}}\right) \\
\cdot(J(J+1)-9)-D_{\mathrm{v}}(J(J+1)-9)^{2}
\end{aligned}
$$

It is difficult to give general analytical solutions to the two cubic equations resulting from matrix $\mathbf{1 0}$ and its antisymmetric counterpart and it is easiest to resort to numerical methods. However for the particular case were $J<3$ it may be seen that both $a^{\prime}$ and $b^{\prime}$ are zero. This simplification allows analytical expressions for the frequencies of the four $|l|=1$ transitions to be obtained as the solutions of the symmetric and antisymmetric determinants. Thus for the $J=1 \rightarrow 2$ transition we obtain

$$
\begin{aligned}
& v_{\mathrm{a}^{ \pm}}=2(J+1)\left(B_{\mathrm{v}}-\gamma_{11^{\prime}}+2 D_{\mathrm{v}}\right) \\
& -4 D_{\mathrm{v}}(J+1)^{3}-2(J+1) q_{\mathrm{t}^{\prime}} / 4 \\
& \pm 1 / 2\left\{\left[\left(q_{\mathrm{t}^{\prime}} / 2-2 \gamma_{1 \mathrm{l}^{\prime}}\right) J(J+1)\right.\right. \\
& \left.-2\left(g_{\mathrm{tt}}-2 g_{\mathrm{tt}}-\gamma_{11^{\prime}}\right)\right]^{2} \\
& +4\left[\left((\sqrt{2})^{-1} q_{\mathrm{t}}-\sqrt{\mathbf{2}} r_{\mathrm{tt}^{\prime}}\right) J(J+1)\right. \\
& \left.\left.-\sqrt{2} r_{\mathrm{tt}^{\prime}}\right]^{2}\right\}_{(J+1)}^{1 / 2} \\
& \pm 1 / 2\{\ldots\}_{J}^{1 / 2} \\
& v_{\mathrm{s}}^{ \pm}=2(J+1)\left(B_{\mathrm{v}}-\gamma_{\mathrm{Il}^{\prime}}+2 D_{\mathrm{v}}\right) \\
& -4 D_{\mathrm{v}}(J+1)^{3}+2(J+1) q_{\mathrm{t}^{\prime}} / 4 \\
& \pm 1 / 2\left\{\left[\left(q_{\mathrm{t}^{\prime}} / 2+2 \gamma_{11^{\prime}}\right) J(J+1)\right.\right. \\
& \left.+2\left(g_{\mathrm{tt}^{\prime}}-2 g_{\mathrm{tt}}-\gamma_{11^{\prime}}\right)\right]^{2} \\
& +4\left[\left((\sqrt{2})^{-1} q_{\mathrm{t}}-\sqrt{2} r_{\mathrm{tt}^{\prime}}^{1}\right) J(J+1)\right. \\
& \left.\left.+\sqrt{2} r_{\mathrm{tt}}^{0}\right]^{2}\right\}_{(J+1)}^{1 / 2} \\
& \mp 1 / 2\{\ldots\}_{J},
\end{aligned}
$$

where the contents of the second empty bracket is identical with that of the first.

From these expressions it is clear that we expect two doublets centred $1 / 2 q_{t^{\prime}} \times 2(J+1)$ apart each with a doublet separation which is dependent on $q_{\mathrm{t}}, q_{\mathrm{t}^{\prime}}, \gamma_{\mathrm{ll}^{\prime}}, r_{\mathrm{tt}^{\prime}}^{1}, r_{\mathrm{tt}^{\prime}}^{0}, g_{\mathrm{tt}}$, and $g_{\mathrm{tt}^{\prime}}$.

From Eqs. (11) and (12) we can extract two sum rules

$$
\begin{gathered}
(1 / 2)\left(v_{\mathrm{s}^{+}}+v_{\mathrm{s}^{-}}\right)^{*}-1 / 2\left(v_{\mathrm{a}}++v_{\mathrm{a}}^{-}\right)^{*}=(1 / 2) q_{\mathrm{t}^{\prime}}, \\
(1 / 4)\left(v_{\mathrm{s}^{+}}+v_{\mathrm{s}^{-}}+v_{\mathrm{a}}++v_{\mathrm{a}}{ }^{-}\right) \\
=\left(B_{\mathrm{v}}-\gamma_{11^{\prime}}\right)+2 D_{\mathrm{v}}-2 D_{\mathrm{v}}(J+1)^{2} .
\end{gathered}
$$

Although Eqs. (11) and (12) are only strictly applicable to the $J=1 \rightarrow 2$ from the experimental observations it appears that the sum rules (Eqs. 
(13), (14)) are also valid for the $J=2 \rightarrow 3$ transition and hence probably useful for other low- $J$ transitions.

\section{Observations}

\section{The Laser Effects}

The 9.4 $\mu \mathrm{m} \mathrm{P}(14) \mathrm{CO}_{2}$ laser line was observed to produce a decrease in intensity in the ground state and an increase in the $\left(1,0^{0}, 0^{0}\right)$ excited state $J=0 \rightarrow 1,1 \rightarrow 2$ and $2 \rightarrow 3$ rotational transitions. At a pressure of $\cong 100 \mathrm{mT}$ Torr for the $J=2 \rightarrow 3$ transition a decrease of $\cong 3 \%$ in the ground state and an increase of $\cong 300 \%$ in the excited state were observed. The magnitude of the effect was observed to decrease with decreasing pressure. Signals of similar magnitude were observed in the $J=0 \rightarrow 1$ and $J=1 \rightarrow 2$ transitions. The change in intensity produced in the excited state $J=2 \rightarrow 3$ Stark modulated signal under the influence of 4 Watts of $\mathrm{CO}_{2}$-laser radiation is shown in Figure 2.

The change in microwave absorption produced by the laser power is of the same sign as that expected to be produced by an increase in temperature. However, here obviously a change in bulk temperature was not occurring, since to produce the observed ground state change a temperature rise of only $\cong 4{ }^{\circ} \mathrm{C}$ was required whereas in the excited state a rise of $\cong 130^{\circ} \mathrm{C}$ was required. It was reasoned that the effects of laser pumping of a particular ro-vibration transition with $J$ much higher than those of the rotational transitions monitored, were being observed as a general change

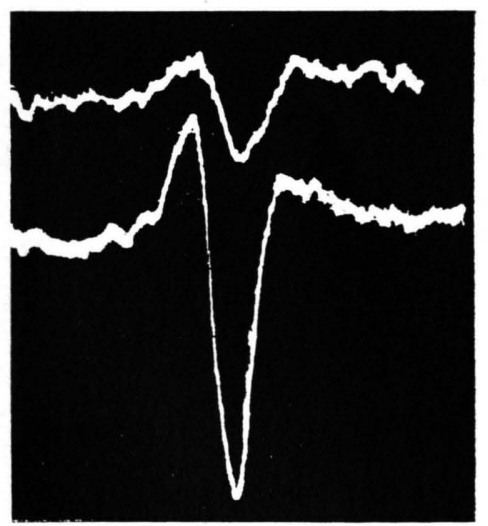

Fig. 2. Increase in absorption intensity produced in the $J=2 \rightarrow 3$ rotational transition of the $\left(1,0^{0}, 0^{0}\right)$ state of DCCF by the $9.4 \mu \mathrm{m}, \mathrm{P}(14) \mathrm{CO}_{2}$ laser line. The upper and lower traces show the observed signals in absence and presence of laser radiation respectively. in the population of the ground and excited states. The mechanism for such an effect would seem to be that the excess population produced in the particular levels of the ground and excited state was spread to the other levels within the vibrational state by successive collisional transitions. Assuming vibrational relaxation to procede relatively slowly, for rotational levels considerably removed from those directly pumped, it would seem that such a mechanism would result in a situation within the vibrational states similar to a new Boltzmann distribution being evolved. Since such a mechanism depends on a large number of collisions this effect would be expected to be more pronounced at higher pressure. In order to determine which ro-vibrational transition of the $\nu_{3}$ band of DCCF was coincident with the laser line a conventional infrared study of this band was carried out. Although the individual ro-vibrational transitions could be resolved, some difficulty was experienced in making an assignment since the band centre was masked by hot-bands and possibly by absorption by residual HCCF. However, a transition was assigned as $\mathrm{R}(9)$ at $1052.25 \pm 0.1$ $\mathrm{cm}^{-1}$ close to the frequency of the $\mathrm{P}(14) \mathrm{CO}_{2}$-laser line $\left(1052.1955 \mathrm{~cm}^{-1}\right)$. Since this assignment was made by combination differences and the spectrum was not too clean this assignment could be out in either direction by one $J$ unit.

Thus from this evidence it appeared that very strong double resonance effects could be expected in ground state transitions with $J$ in the region of 9 . Using the mm-wave equipment already described the three ground states transitions $J=5 \rightarrow 6$, $8 \rightarrow 9$ and $11 \rightarrow 12$ could be simultaneously monitored as the second, third and fourth harmonic of a fundamental frequency of $52.4 \mathrm{GHz}$. In the event of the laser pumping the $R(9)$ infrared transition strong double resonance effects would be expected on the $J=8 \rightarrow 9$ transition and appreciable effects would also be expected on the other two transitions. After optimization of the system a weak double resonance signal was observed on the $J=5 \rightarrow 6$ transition and none at all on the other two, much less intense, transitions. The effect observed on the $J=5 \rightarrow 6$ transition displayed similar characteristics to those of the lower $J$ transitions. Thus it appears that the explanation involving the $v_{3}, \mathrm{R}(9)$ transition is incorrect.

Since the observed effect was specific to the laser line used and was not present in HCCF, absorption 
of laser radiation by DCCF, or at least a deuterated inpurity must be involved. With such a simple molecular system there are only a very limited number of possibilities. Considerable effort was devoted to devising alternative explanations for the observations and these will be summarized below.

1. Absorption occurs in other band e.g. hot band. No appreciable signal on rotational transitions other than those of ground state and $\left(1,0^{0}, 0^{0}\right)$ was observed.

2. Absorption occurs in an impurity and the effect in DCCF was produced by collisional transfer. This mechanism seems to be a definite possibility but the properties of such an impurity were determined to be so similar to DCCF itself that we are at a loss for a suitable candidate. The impurity must be easily deuterated and have an absorption band in the $10 \mu \mathrm{m}$ region when deuterated. From experiments with DCCF samples held at low temperatures it was determined that the signal producing agent had a similar vapour pressure to DCCF. This fact rules out most of the possible impurities arising from the synthesis and the remaining most likely possibilities. DCCD, DCCH and $\mathrm{D}_{2} \mathrm{O}, \mathrm{HDO}$ were found not to be responsible for this effect. An observation which lends some support to this mechanism was that in mixtures of HCCF and DCCF, signals were also observed in low $J$ ground and $\left(1,0^{0}, 0^{0}\right)$ excited state transitions of HCCF. A sample of HCCF which had had no contact with $\mathrm{D}_{2} \mathrm{O}$ did not produce these signals.

At the present time we are unable to determine whether the observed laser-induced signals arise by an absorption by DCCF itself or via absorption by an easily deuterable impurity. Naturally, in principle this question may be resolved by either purification of the DCCF sample by gas chromatography, or by producing a sample over another synthetic route. Both possibilities require considerably chemical manipulation which are not possible in our laboratory at the present time.

Thus whilst we are unable to definitely determine the mechanism of the effect, the laser induced enhancement of the intensity of the excited state transition (Fig. 2) was appreciable, and for studies of high energy excited states brings a welcome increase in absorption intensity over a considerable range of $J$.

\section{The Microwave Spectrum}

The transition frequencies measured for the various vibrational states of fluoroacetylene divided by $2(J+1)$ are shown in Table 1 .

\section{Ground State}

Ground state transitions up to maximum frequency of $209.6 \mathrm{GHz}$ were measured for the D-species and up to $194.1 \mathrm{GHz}$ for the $\mathrm{H}$-species of fluoroacetylene. These measurements allowed both $B_{0}$ and $D_{J}$ to be accurately determined for both species and the calculated values for these constant are shown in Table 2 . For the four ${ }^{13} \mathrm{C}$ species only transitions up to $J=2 \rightarrow 3$ were measured and in calculating the $B_{0}$-constants for these species the values of $D_{J}$ determined for the ${ }^{12} \mathrm{C}$ species were assumed.

\section{State $\left(1,0^{0}, 0^{0}\right)$}

Transitions up to $J=2 \rightarrow 3$ were measured for both HCCF and DCCF. In DCCF the increase in absorption intensity produced by the laser radiation, which has already been discussed, was an aid in making these measurements. The constant $B_{0}$ and an approximately value for $D_{\mathrm{v}}$ was determined from these measurements.

States $v_{\mathrm{t}}=1, v_{\mathrm{t}}=3 ;$ States $\left(0,1^{1}, 0^{0}\right),\left(0,0^{0}, 1^{1}\right)$ and $\left(0,0^{0}, 3^{1}\right)$

Rotational transitions $J \rightarrow J+1$ were measured in these vibrational states up to $J=7 \rightarrow 8$ in HCCF and $J=8 \rightarrow 9$ in DCCF (Table 1). Using these frequencies together with Eq. (5), $B_{\text {eff }}, D_{\mathrm{v}}$ and an approximate value for $q_{\mathrm{t}}$ may be calculated. The values of $B_{\text {eff }}$ and $D_{\mathrm{v}}$ so calculated are shown in Table 2.

Direct $l$-type doubling transitions $J \rightarrow J$ were also measured in the state $\left(0,1^{1}, 0^{0}\right)$ and $\left(0,0^{0}, 1^{1}\right)$ up to a maximum of $J=31$. The experimental frequencies of these transitions are shown in Table 3, and the values of $q_{\mathrm{t}}{ }^{0}$ and $q_{\mathrm{t}}{ }^{1}$ obtained by a least square fit to Eq. (6) are shown in Table 2. Higher order terms than those shown in Eq. (6) were found not to make significant contributions to the frequency of the direct $l$-type doubling transitions. It should be pointed out that the l-type doubling constants determined in this work are considerably different from those determined earlier [4]. Since these early measurements invariably yielded constants which were too small it appears 
Table 1. Experimental frequencies in $\mathrm{MHz}$ divided by $2(J+1)$.

\begin{tabular}{|c|c|c|c|c|c|c|c|}
\hline & State & & $J=0 \rightarrow 1$ & $J=1 \rightarrow 2$ & $J=2 \rightarrow 3$ & $J=4 \rightarrow 5$ & $J=7 \rightarrow 8$ \\
\hline \multirow[t]{10}{*}{ HCCF } & Ground $^{\mathrm{a}}$ & & 9706.171 & 9706.151 & 9706.125 & 9706.22 & 9705.731 \\
\hline & $\left(0,1^{1}, 0^{0}\right)$ & $|l|=1$ & $\overline{-}$ & $\begin{array}{l}9721.387 \\
9708.572\end{array}$ & $\begin{array}{l}9721.336 \\
9708.535\end{array}$ & $\begin{array}{l}9721.254 \\
9708.422\end{array}$ & $\begin{array}{l}9720.965 \\
9708.136\end{array}$ \\
\hline & $\left(0,2^{0,2}, 0^{0}\right)$ & $\left\{\begin{array}{l}l \\
l \mid=\mathbf{0}\end{array}\right.$ & $\begin{array}{c}9723.27 \\
-\end{array}$ & $\begin{array}{c}9723.283 \\
-\end{array}$ & $\begin{array}{l}9723.291 \\
9722.657\end{array}$ & - & $\overline{-}$ \\
\hline & $\left(0,0^{0}, 1^{1}\right)$ & $|l|=1$ & - & $\begin{array}{l}9743.488 \\
9724.136\end{array}$ & $\begin{array}{l}9743.440 \\
9724.098\end{array}$ & $\begin{array}{l}9743.328 \\
9723.984\end{array}$ & $\begin{array}{l}9743.051 \\
9723.696\end{array}$ \\
\hline & $\left(0,0^{0}, 2^{0,2}\right)$ & $\left\{\begin{array}{l}l \\
l \\
l \\
l\end{array} \mid=2\right.$ & $\begin{array}{c}9760.58 \\
- \\
-\end{array}$ & $\begin{array}{c}9760.525 \\
- \\
-\end{array}$ & $\begin{array}{l}9760.448 \\
9761.06\end{array}$ & $\begin{array}{l}9760.147 \\
9760.928 \\
\mathbf{9 7 6 1 . 1 9 0}\end{array}$ & $\begin{array}{l}9759.408 \\
9760.629 \\
9761.320\end{array}$ \\
\hline & $\left(0,0^{0}, 3^{1}\right)$ & $|l|=1$ & - & $\begin{array}{l}9806.095 \\
9767.654\end{array}$ & $\begin{array}{l}9806.048 \\
9767.637\end{array}$ & - & - \\
\hline & $\left(0,0^{0}, 4^{0,2}\right)$ & $\left\{\mid \begin{array}{l}l \\
l \mid=0 \\
=0\end{array}\right.$ & $\overline{-}$ & $\begin{array}{c}9812.22 \\
-\end{array}$ & $\begin{array}{l}9812.016 \\
9812.952\end{array}$ & - & - \\
\hline & $\left(0,(1,1)^{0,2}\right)$ & $\left\{\begin{array}{l}l \\
l \\
l \\
l\end{array} \mid=0\right.$ & $\begin{array}{c}9742.76 \\
9741.97 \\
-\end{array}$ & $\begin{array}{c}9742.75 \\
9741.97 \\
-\end{array}$ & $\begin{array}{l}9742.727 \\
9741.985 \\
9742.350\end{array}$ & $\begin{array}{l}- \\
- \\
-\end{array}$ & $\begin{array}{l}- \\
-\end{array}$ \\
\hline & $\left(0,(1,2)^{1}\right)$ & $|l|=1$ & $\begin{array}{l}- \\
- \\
-\end{array}$ & $\begin{array}{l}9755.167 \\
9762.311 \\
9776.604 \\
9782.318\end{array}$ & $\begin{array}{l}9755.156 \\
9762.285 \\
9776.608 \\
9782.308\end{array}$ & $\begin{array}{l}- \\
- \\
-\end{array}$ & $\begin{array}{l}- \\
- \\
-\end{array}$ \\
\hline & $\left(1,0^{0}, 0^{0}\right)$ & & 9669.05 & 9669.037 & 9669.008 & - & - \\
\hline \multirow[t]{13}{*}{ DCCF } & & & & & & $J=5 \rightarrow 6$ & $J=8 \rightarrow 9$ \\
\hline & Ground ${ }^{b}$ & & 8736.036 & 8736.000 & 8735.982 & 8735.835 & 8735.589 \\
\hline & $\left(0,1^{1}, 0\right)$ & $|l|=1$ & $\begin{array}{l}- \\
-\end{array}$ & $\begin{array}{l}8757.872 \\
8744.360\end{array}$ & $\begin{array}{l}8757.846 \\
8744.328\end{array}$ & $\begin{array}{l}8757.69 \\
8744.17\end{array}$ & $\begin{array}{l}- \\
-\end{array}$ \\
\hline & $\left(0,2^{0,2}, 0\right)$ & $\left\{\mid \begin{array}{l}l \\
l \mid=\mathbf{0}\end{array}\right.$ & $\begin{array}{c}8766.420 \\
-\end{array}$ & $\begin{array}{c}8766.410 \\
-\end{array}$ & $\begin{array}{l}8766.385 \\
8765.507\end{array}$ & - & - \\
\hline & $\left(0,3^{1}, 0\right)$ & $|l|=1$ & - & $\begin{array}{c}8794.8 \\
-\end{array}$ & $\begin{array}{c}8794.76 \\
-\end{array}$ & - & - \\
\hline & $\left(0,0^{0}, 1^{1}\right)$ & $|l|=1$ & $\overline{-}$ & $\begin{array}{l}8768.402 \\
8752.715\end{array}$ & $\begin{array}{l}8768.376 \\
8752.686\end{array}$ & $\begin{array}{l}8768.228 \\
8752.530\end{array}$ & $\begin{array}{l}8767.978 \\
8752.288\end{array}$ \\
\hline & $\left(0,0^{0}, 2^{0,2}\right)$ & $\left\{\begin{array}{l}l \\
l \\
l \\
l\end{array} \mid=2\right.$ & $\begin{array}{c}8784.020 \\
- \\
-\end{array}$ & $\begin{array}{c}8783.960 \\
- \\
-\end{array}$ & $\begin{array}{l}8783.925 \\
8784.668\end{array}$ & $\begin{array}{l}8783.603 \\
8784.708 \\
8784.563\end{array}$ & $\begin{array}{l}- \\
-\end{array}$ \\
\hline & $\left(0,0^{0}, 3^{1}\right)$ & $|l|=1$ & $\overline{-}$ & $\begin{array}{l}8822.60 \\
8791.39\end{array}$ & $\begin{array}{l}8822.556 \\
8791.366\end{array}$ & $\overline{-}$ & $\overline{-}$ \\
\hline & $\left(0,0^{0}, 4^{0,2}\right)$ & $\left\{\mid \begin{array}{l}l \\
l \mid=0\end{array}\right.$ & - & $\begin{array}{c}8828.916 \\
-\end{array}$ & $\begin{array}{l}8828.838 \\
8829.636\end{array}$ & - & - \\
\hline & $\left(0,(1,1)^{0,2}\right)$ & 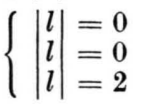 & $\begin{array}{c}8775.396 \\
8775.786 \\
-\end{array}$ & $\begin{array}{c}8775.402 \\
8775.737 \\
-\end{array}$ & $\begin{array}{l}8775.418 \\
8775.747 \\
8775.500\end{array}$ & $\begin{array}{l}- \\
- \\
-\end{array}$ & $\frac{-}{-}$ \\
\hline & $\left(0,(2,1)^{1}\right)$ & $|l|=1$ & $\begin{array}{l}- \\
- \\
-\end{array}$ & $\begin{array}{l}8786.14 \\
8786.63 \\
8792.86 \\
8795.56\end{array}$ & $\begin{array}{l}8786.112 \\
8786.628 \\
8792.904 \\
8795.544\end{array}$ & $\begin{array}{l}- \\
- \\
-\end{array}$ & $\begin{array}{l}- \\
- \\
-\end{array}$ \\
\hline & $\left(0,(1,2)^{1}\right)$ & $|l|=1$ & $\begin{array}{l}- \\
- \\
-\end{array}$ & $\begin{array}{l}8789.69 \\
8796.47 \\
8801.88 \\
8808.67\end{array}$ & $\begin{array}{l}8789.682 \\
8796.432 \\
8801.856 \\
8808.678\end{array}$ & $\begin{array}{l}- \\
- \\
-\end{array}$ & $\begin{array}{l}- \\
- \\
-\end{array}$ \\
\hline & $\left(1,0^{0}, 0^{0}\right)$ & & 8704.860 & 8704.803 & 8704.795 & - & - \\
\hline
\end{tabular}

a $J=8 \rightarrow 9=9705.593 ; J=9 \rightarrow 10=9705.504 ;$ b $J=9 \rightarrow 10=8735.472 ; J=11 \rightarrow 12=8735.253$. 
Table $1 \mathrm{a}$.

\begin{tabular}{|c|c|c|c|c|c|c|c|}
\hline & \multicolumn{2}{|l|}{$\mathrm{H}^{13} \mathrm{CCF}$} & \multicolumn{2}{|l|}{$\mathrm{HC}^{13} \mathrm{CF}$} & \multirow{2}{*}{$\frac{\mathrm{D}^{13} \mathrm{CCF}}{J=2 \rightarrow 3}$} & \multicolumn{2}{|l|}{$\mathrm{DC}^{13} \mathrm{CF}$} \\
\hline & $J=1 \rightarrow 2$ & $J=2 \rightarrow 3$ & $J=1 \rightarrow 2$ & $J=2 \rightarrow 3$ & & $J=1 \rightarrow 2$ & $J=2 \rightarrow 3$ \\
\hline Ground & 9373.912 & 9373.887 & 9700.621 & 9700.580 & 8486.160 & 8733.858 & 8733.840 \\
\hline$\left(0,1^{1}, 0^{0}\right)$ & $\overline{-}$ & $\begin{array}{l}9375.321 \\
9387.291\end{array}$ & - & $\begin{array}{l}9702.968 \\
9715.746\end{array}$ & $\begin{array}{l}8493.192 \\
8506.062\end{array}$ & $\begin{array}{l}- \\
-\end{array}$ & - \\
\hline$\left(0,0^{0}, 1^{1}\right)$ & - & $\begin{array}{l}9391.535 \\
9409.673\end{array}$ & - & $\begin{array}{l}9716.973 \\
9736.873\end{array}$ & $\begin{array}{l}8502.684 \\
8517.546\end{array}$ & - & - \\
\hline
\end{tabular}

Table 2. Spectroscopic Constants.

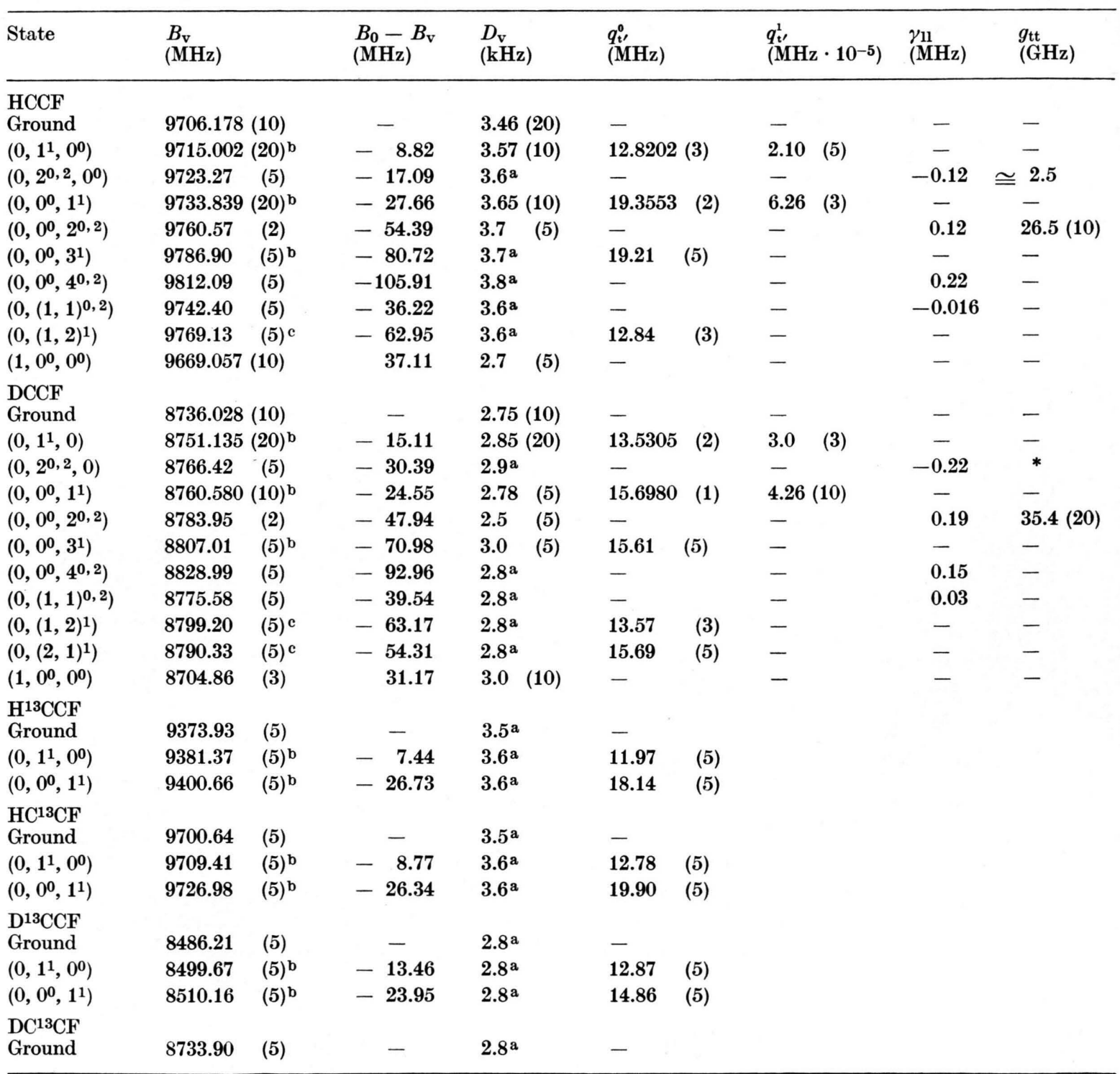

a assumed, b $B_{\text {eff }}=\left(B_{\mathrm{v}}+\gamma_{11}\right)$, c $B_{\text {eff }}=\left(B_{\mathrm{v}}-\gamma_{11^{\prime}}\right)$, ${ }^{*}$ see text. 
Table 3. Direct $l$-type doubling transitions $J \rightarrow J(\mathrm{MHz})$.

\begin{tabular}{lrrr}
\hline & $J$ & HCCF & DCCF \\
\hline State $\left(0,0^{0}, 1^{1}\right)$ & 17 & - & 4799.61 \\
& 18 & 6612.33 & 5363.71 \\
& 19 & - & 5959.06 \\
& 20 & 8118.30 & - \\
21 & 8928.84 & 7243.40 \\
& 22 & 9777.70 & 7932.36 \\
& 23 & 10664.98 & - \\
& 24 & 11590.56 & 9403.46 \\
& 25 & 12554.52 & 10185.69 \\
& 26 & 13556.61 & 10999.02 \\
& 27 & 14594.89 & 11843.38 \\
& 28 & - & 12718.75 \\
& 29 & 16791.82 & 13624.98 \\
& 30 & - & 14562.33 \\
& 31 & - & 15530.54 \\
& 22 & - & 6838.97 \\
& 23 & 7070.33 & 7459.82 \\
& 24 & 7684.65 & 8107.56 \\
& 25 & 8324.27 & 8782.28 \\
& 26 & 8989.34 & - \\
27 & 9680.16 & 10212.06 \\
& 28 & 10396.16 & - \\
& 29 & - & 11749.09 \\
& 30 & - & 12557.81 \\
\hline 0 & & & \\
\hline
\end{tabular}

that experimental error was introduced probably through insufficiently accurate zero-basing of the Stark voltage used.

States with $v_{\mathrm{t}}=2 ;\left(0,0^{0}, 2^{0,2}\right)$

Rotational transitions up to $J=7 \rightarrow 8$ and $J=5 \rightarrow 6$ were measured for HCCF and DCCF respectively. In the case of $\mathrm{HCCF}$ the data was sufficient to allow a complete assignment to be made. As may be seen from Eqs. (1) and (2) we expect three transitions, $v_{\mathrm{a}}$ identifiable with one of the $|l|=2$ transitions and two $v_{\mathrm{s}}$ transition identifiable with one $|l|=2$ and one $|l|=0$ transition. These latter two transitions are mutually perturbed via the matrix elements

$$
\left\langle v_{\mathrm{t}}, l=0|H| v_{\mathrm{t}}, l=2\right\rangle .
$$

From the values of $v^{*}$ in Table 1 it can be seen that the higher frequency $|l|=2$ transitions displays an anomalous shift to high frequency with increasing $J$ and that the $|l|=0$ transition displays a stronger shift to lower frequency than one would expect from centrifugal distortion alone. Thus we can identify the higher frequency $|l|=2$ transition as the symmetric solution with the positive square root, i.e. $v_{\mathrm{s}}{ }^{+}$and the $|l|=0$ as $v_{\mathrm{s}}{ }^{-}$. Consequently the remaining set of transitions with $|l|=2$ is $v_{\mathrm{a}}$.
Once this assignment has been made, using the Eqs. (3) and (4) the constants $B_{\mathrm{v}}, D_{\mathrm{v}}$ and $\gamma_{11}$ may be determined and are shown in Table 2. Once these constants have been determined, the anharmonic constant $g_{\mathrm{tt}}$ which contributes to the energy separation between the states with $|l|=\mathbf{2}$ and $|i|=0$, may be evaluated by use of Equation (2). A value of $\Delta_{2}{ }^{0}=+16.8 \mathrm{GHz}$ reproduced the separation between the $\nu_{\mathrm{s}}{ }^{+}$and $v_{\mathrm{s}}{ }^{-}$components of the $J=4 \rightarrow 5$ and $7 \rightarrow 8$ transitions to within the experimental error. Thus from the known values of $B_{5}, \gamma_{55}$ and $D_{5}$ we calculate $g_{55}=+26.5 \pm 1.0 \mathrm{GHz}$.

For DCCF the situation was almost identical. Although the two $|l|=2$ transitions were resolved in only one transition, $J=5 \rightarrow 6$, it is obvious that here again the higher frequency $|l|=2$ transition is also perturbed towards higher frequency. Thus the transitions may be assigned as in HCCF. The constants calculable with the sum rules (Eqs. (3) and (4)) are shown in Table 2. From the measured separation between $v_{\mathrm{s}}{ }^{+}$and $v_{\mathrm{s}}{ }^{-}$in the $J=5 \rightarrow 6$ transition we arrive at values of $\Delta_{2}{ }^{0}=+26.5 \mathrm{GHz}$, $g_{55} \cong+35.4 \pm 2.0 \mathrm{GHz}$.

The calculation of $g_{55}$ was directly dependent on the separation between $v_{\mathrm{s}}{ }^{+}$and $v_{\mathrm{s}}{ }^{-}$, where $v_{\mathrm{s}}{ }^{-}$was identifiable with $|l|=0$. Consequently any effect which disturbs the relative positions of these lines, such as Fermi resonance, will be incorporated into the effective values of $g_{55}$ and $\gamma_{55}$. The error margins given on these quantities here simply reflects the fit to the microwave data and as such must be treated with caution. From consideration of Fig. 1 it may bee seen that for HCCF such effects are unlikely, but for DCCF a weak interaction with $\left(0,(1,1)^{0,2}\right)$ which is $\sim 80 \mathrm{~cm}^{-1}$ away may possibly occur.

$\left(0,2^{0,2}, 0\right)$

In principle the situation here is similar to the $\left(0,0,2^{0,2}\right)$ state, but since $v_{4}$ is considerably higher in frequency than $v_{5}$, the rotational transitions in this state were much weaker and were not observed in the relatively insensitive $\mathrm{mm}$-wave spectrometer used. However from the data available (Table 1) it may be seen that the situation here was quite different from that of the low-frequency bending mode. Firstly the transition identifiable with $|l|=2$ lies to the low frequency side of that with $|l|=0$. Secondly for HCCF the $|l|=0$ transitions display a $J$-dependence opposite to that expected 
from distortion effects and it appears that in this case we must consider these transitions to be described by taking the positive square root in Eq. (2), i.e. $(l=0)=v_{\mathrm{s}}{ }^{+}$.

For HCCF since the $|l|=2$ doublet was not resolved in the $J=2 \rightarrow 3$ transition the single frequency must be used for both $\nu_{\mathrm{s}^{-}}$and $\nu_{\mathrm{a}}$. With an assumed distortion constant of $D_{\mathrm{v}}=3.6 \mathrm{kHz}$, from this transition we calculated $\gamma_{44}=-0.16$ and $B_{\mathrm{v}}=9723.35 \mathrm{MHz}$ using Eqs. (3) and (4). This value for $B_{\mathrm{v}}$ was considerably larger than that determinable from $J=0 \rightarrow 1,|l|=0$ transition $\left(v^{*}=9723.27\right)$ and in $\left(0,0^{0}, 2^{0,2}\right)$ for HCCF and DCCF and $\left(0,2^{0,2}, 0\right)$ for DCCF these two quantities are very similar. The difference between these two values in the present case was almost certainly caused by the anomalous shift in frequency with $J$ of the $|l|=0$ transition (here $v_{\mathrm{s}}{ }^{+}$) not being compensated by that of the $\nu_{\mathrm{s}}{ }^{-}$transition which was not resolved here. For the same reason the $\gamma_{44}$ calculated from the $J=2 \rightarrow 3$ transition alone is probably too large. Consequently the constants produced by fixing $B_{\mathrm{v}}$ at $v^{*}$ for the $J=0 \rightarrow 1$, $|l|=0$ transition and recalculating $\gamma_{44}$ are probably more meaningful. The constants obtained are shown in Table 2. Using these constants we arrive at values of $\Delta_{2}{ }^{0} \cong-7.0 \mathrm{GHz}$ and $g_{44} \cong+2.5 \mathrm{GHz}$.

In DCCF the $|l|=2$ transition was also observed to the low frequency side of the $|l|=0$ transition for $J=2 \rightarrow 3$. However no anomalous behaviour of the $|l|=0$ transitions was observed, i.e. $v^{*}$ decreases with increasing $J$. Thus in this case an exact assignment was not possible. In the application of Eq. (3) the assignment is of no consequence and in this case since the two $|l|=2$ transitions were not resolved there is no difficulty in applying Equation (4). Using these two equations with an assumed value of $D_{\mathrm{v}}$ the constants $D_{\mathrm{v}}$ and $\gamma_{11}$ were calculated.

In the $\left(0,0^{0}, 2^{0,2}\right)$ of fluoro, chloro [5] and bromoacetylene [6] the $|l|=2$ transitions were observed to the high frequency side of the $|l|=0$ transition whereas here in the $\left(0,2^{0,2}, 0^{0}\right)$ state of both HCCF and DCCF the situation was reversed. One is tempted to seek some form of coupling to explain this situation, but as may be seen from Fig. 1 the environment of $\left(0,2^{0,2}, 0\right)$ in HCCF and DCCF are quite different. In both cases the nearest state is approximately $100 \mathrm{~cm}^{-1}$ away. For HCCF a Fermi resonance between $\left(0,2^{0}, 0\right)$ and $\left(1,0^{0}, 0^{0}\right)$ might be expected and indeed such an interaction has been the subject of an infrared study [12]. It was concluded from this investigation that such an interaction was weak, and more to the point such an interaction would not explain the observations. This is because mixing between $\left(0,2^{0}, 0\right)$ and $(1,0,0)$ would tend to reduce the observed effective $v^{*}$ for the $|l|=0$ transition from its unperturbed value and a mechanism producing the opposite effect is required here. At the present time we have no realistic suggestion to explain these observations.

$v_{\mathrm{t}}=4,\left(0,0^{0}, 4^{0,2}\right)$

Since this state lies near $1460 \mathrm{~cm}^{-1}$ for both HCCF and DCCF the rotational transitions were very weak and the data obtained was somewhat limited. For the two transitions measured for both molecular species it appears that the $|l|=0$ transitions display a larger negative shift with increasing $J$ than might be expected from distortion effects. Thus it seems that in both cases we must assign $(|l|=0)=v_{\mathrm{s}}{ }^{-}$and $(|l|=2)=v_{\mathrm{a}}, \nu_{\mathrm{s}}{ }^{+}$. Using the sum rules, Eqs. (3) and (4), with an assumed value of $D_{\mathrm{v}}, B_{\mathrm{v}}$ and $\gamma_{11}$ were calculated (Table 2).

\section{Combination States}

$v_{\mathrm{t}}=1, v_{\mathrm{t}}{ }^{\prime}=1 ;\left(0,(1,1)^{0,2}\right)$

In this state the possibility exists of four components being observed for every $J>2$. However for both isotopic species of fluoroacetylene the highest $J$ transition measured was $J=2 \rightarrow 3$ and in this transition the $|l|=2$ transition were not resolved. There is unfortunately insufficient data to make complete assignments of the components and only the first of the sum rules (Eq. (7)) given for this state is independent of the assignment. In HCCF it appears that both $|l|=0$ transitions display a positive shift relative to that expect from centrifugal distortion. In DCCF the same can be said for the lower frequency $|l|=0$ component, but for the other the situation is not clear. Thus it seems that in HCCF both $|l|=0$ components are conducive with taking a positive square root (i.e. $\nu^{+}$) and this is probably the case for DCCF. It was not possible to assign the transitions as symmetric or antisymmetric. Using the first sum rule and assumed values of $D_{J}$ the rotational constants shown in Table 2 were calculated. With the assignment given above and the third approximate sum rule (Eq. (9)) a value for $\gamma_{11}$ was derived from 
the $J=2 \rightarrow 3$ data. As may be seen from Table 2 the value obtained for this constant in this state is very much smaller than those obtained in the $\left(0,0^{0}, 2^{0,2}\right)$ and $\left(0,2^{0,2}, 0^{0}\right)$ states. This is in-keeping with the fact that $\gamma_{44}$ and $\gamma_{55}$ for these two latter states were determined to have similar magnitudes but opposite signs, thus leading to near cancellation in the combination state.

In order to be able to apply the Eq. (8) the assignment into symmetric and antisymmetric species is necessary. Since this was not possible the magnitude but not the sign of the quantities determinable by use of this sum rule may be calculated.

From the $J=0 \rightarrow 1$ transition we have simply

$$
v^{*}=\left(B_{\mathrm{v}}-\gamma_{11}\right)-2 D_{\mathrm{v}} \pm r_{\mathrm{tt}^{\prime}}^{1}
$$

thus $\left|r_{\mathrm{tt}^{\prime}}^{1}\right|$ may be determined directly from this transition. Since the $|l|=2$ components were not resolved applying the sum rule to the other two transitions measured is essentially the same as considering the difference between $v^{*}$ for the two $|l|=0$ components. Thus we have

$$
\left|\Delta v^{*}(l=0)\right|=2\left(r_{\mathrm{tt}^{\prime}}^{1}-2 \varrho_{\mathrm{tt}^{\prime}}\right)-4(J+1)^{2} \varrho_{\mathrm{tt}^{\prime}} .
$$

The terms in $\varrho$ were found to be of negligible importance in the other states treated, but here they have an apparently detectable effect since a consistent decrease in $\Delta v^{*}(l=0)$ was observed in both HCCF and DCCF with increasing $J$. Since the sign of $\left|r_{\mathrm{tt}^{\prime}}^{1}\right|$ was not fixed all that may be said for $\varrho$ is that it has the opposite sign from $r_{\mathrm{tt}^{\prime}}^{1}$. For HCCF $\left|r_{\mathrm{tt}^{\prime}}^{1}\right|=0.39 \mathrm{MHz},\left|\varrho_{\mathrm{tt}^{\prime}}\right| \cong 1 \mathrm{kHz}$ and for DCCF $\left|r_{\mathrm{tt}^{\prime}}^{1}\right|=0.19 \mathrm{MHz}$ and $\left|\varrho_{\mathrm{tt}^{\prime}}\right| \cong 2 \mathrm{kHz}$ were determined.

In principle it was possible to estimate the values of $g_{4}, 5$ using expressions given in [1] but because of the uncertainty in sign of some of the constants it is perhaps better to postpone such a calculation until more data is available.

$v_{\mathrm{t}}=2, v_{\mathrm{t}^{\prime}}=1 ;\left(0,(2,1)^{1}\right),\left(0,(1,2)^{1}\right)$

Transitions of the state $\left(0,(1,2)^{1}\right)$ were measured for both HCCF and DCCF, but only in the case of DCCF were transitions of the state $\left(0,(2,1)^{1}\right)$ observable, since in HCCF this state lies over $1600 \mathrm{~cm}^{-1}$. For rotational transition below $J=3$ we expect four $|l|=1$ components to be observed in this state. In all three cases four lines were measured, positioned roughly symmetrically about the centre frequencies calculated using the $\alpha$-constants obtained from other vibrational states. The experimental values of $v^{*}$ for the transitions of this state are shown in Table 1 and are displayed in Figure 3. From the Eq. (13) (and from calculations in which the matrix 10 was diagonalized) we expect two doublets the centres of which are $1 / 2 q_{\mathrm{t}^{\prime}}$ apart, and it can be seen in Fig. 3 that these are in fact present. The value of $q_{t^{\prime}}$ calculated from the doublet separations is shown in Table 2, and it can be seen that these agree very well with those determined from measurements on the relevant singly excited state. Using Eq. (14) and an assumed value of $D_{\mathrm{v}}$ values for $B_{\text {eff }}=\left(B_{\mathrm{v}}-\gamma_{11^{\prime}}\right)$, were calculated and are shown in Table 2 .

The actual interpretation of the observed transition frequencies however presented a more difficult problem. As may be seen from the Eqs. (11) and (12) the splittings of the symmetric and asymmetric doublets is dependent on a large number of constants and the data available here were certainly not sufficient to determine all of these. With the exception of the anharmonic constants $g_{\mathrm{tt}}, g_{\mathrm{tt}^{\prime}}$ and $r_{\mathrm{tt}^{\prime}}^{0}$ values for the other constants were available from the measurements on other states or the constants themselves were small. For $g_{\mathrm{tt}}, g_{\mathrm{tt}^{\prime}}$ and $r_{\mathrm{tt}^{\prime}}^{0}$ a large range of values were possible and as may be seen from Eqs. (11) and (12) the doublet splittings are strongly dependent on the magnitude of these constants. These equations are only accurate for the $J=1 \rightarrow 2$ transition but for the present discussion we will consider them to hold approximately for the $J=2 \rightarrow 3$ transition. If we neglect all other constants except $q_{\mathrm{t}}, q_{\mathrm{t}^{\prime}}, g_{\mathrm{tt}}$ and $g_{\mathrm{tt}^{\prime}}$ and we consider the latter two as combined in a single constant $G=g_{\mathrm{tt}^{\prime}}-2 g_{\mathrm{tt}}$, it can easily be seen that the doublet splittings divided by $2(J+1)$ have a maximum for $G=0$ of approximately

$$
\left(0.5 q_{\mathrm{t}^{\prime}}^{2}+2 q_{\mathrm{t}^{2}}\right)^{1 / 2},
$$

which reduces to zero as $G \rightarrow \infty$. For the $\left(0,(1,2)^{1}\right)$ state of HCCF this maximum is $\cong 28.8 \mathrm{MHz}$ and for the $\left(0,(1,2)^{1}\right)$ and $\left(0,(2,1)^{1}\right)$ states of DCCF is $\cong 24.1 \mathrm{MHz}$ and $\cong 22.1 \mathrm{MHz}$ respectively compared to average observed separations of $\cong 21.5$, $\cong 12.2$ and $\cong 1.5 \mathrm{MHz}$ respectively. The experimental values were all smaller than the maximum possible splittings and it appears at first sight that 

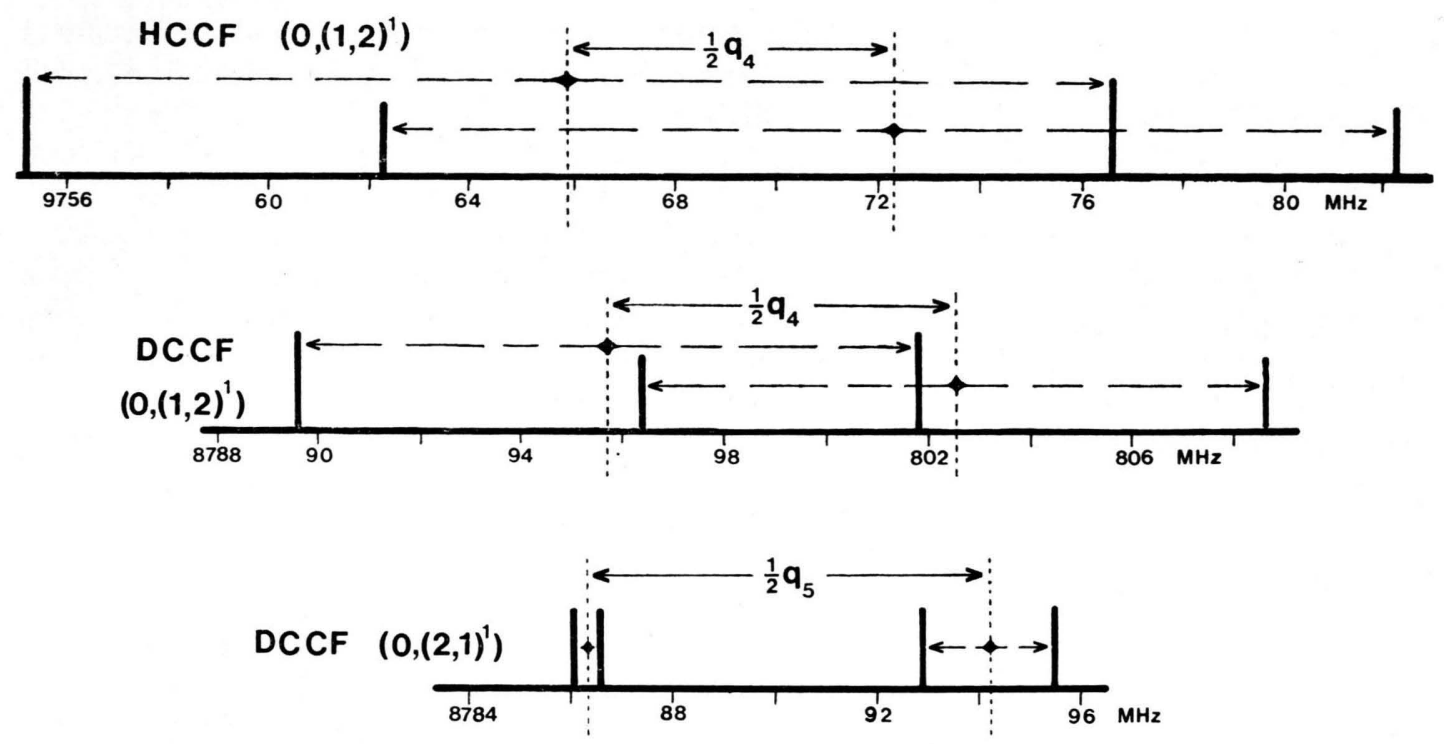

Fig. 3. Diagrammatical representation of the rotational transition frequencies divided by $2(J+1)$ of the states involving $2 v_{\mathrm{t}}+v_{\mathrm{t}^{\prime}}$. The assignment necessary to produce two pairs of transitions with centres separated by $\frac{1}{2} q_{\mathrm{t}^{\prime}}$ are indicated by the broken arrows.

it was only necessary to select appropriate values of $G$ in order to be able to reproduce the observed splittings. However as may be seen from the form of Eqs. (11) and (12) when the cross-terms $G q_{\mathrm{t}^{\prime}} J(J+1)$ are of comparable magnitude to $q_{\mathrm{t}}{ }^{2} J^{2}(J+1)^{2}$, the normal $2(J+1)$ dependence of the transition frequencies of a linear molecule is disturbed. In the case of the $\left(0,(1,2)^{1}\right)$ state of HCCF the observed splitting in the $J=1 \rightarrow 2$ transition was approximately reproduced with $G=-120 \mathrm{MHz}$, but the splitting in the $J=2 \rightarrow 3$ transition was not simultaneously reproduced, even when a procedure involving the diagonalization of matrix 10 was used, i.e. the $2(J+1)$ frequency dependence was lost as under these conditions of near resonance a strong $J$ dependence was introduced. As may be seen from Table 1 our observations on the $J=1 \rightarrow 2$ and $J=2 \rightarrow 3$ transitions lead us to believe that the $2(J+1)$ frequency dependence of the transitions in these combination states was largely preserved. With values of $G$ in excess of $10 \mathrm{GHz}$ the $2(J+1)$ frequency dependence of the calculated rotational transitions frequencies of these states was largely restored. However, for the $\left(0,(1,2)^{1}\right)$ states the calculated splittings were much less than those observed and in the $\left(0,(2,1)^{1}\right)$ state of DCCF the situation was reversed. In principle the calculated splittings for this latter state could be reduced by increasing $G$ to large values. In the range 100 to $5000 \mathrm{GHz}$ the calculated splitting was very insensitive to the magnitude of this constant, and even with the largest value of $G$ used the calculated splittings were still 3 to 4 times larger than those observed.

Throughout this simplified discussion we have assumed that $r_{\mathrm{tt}^{\prime}}^{0}=0$. This is almost certainly not the case and this constant could be of considerable magnitude. Nevertheless the role played by $r_{\mathrm{tt}^{\prime}}^{0}$ in Eqs. (11) and (12) is very similar to $G$ since it generates cross-terms in $(J(J+1))^{1} / 2$. Consequently it appears that inclusion of this constant would not necessarily improve matters, with the possible exception that it would introduce a reduction in the calculated splittings for the $\left(0,(2,1)^{1}\right)$ thus bringing the values closer to those observed. At the present time we have no explanation for this apparent disagreement between experiment and theory and because of the limited data available do not intend to persue this subject further. Whatever the cause of this effect the desirability of making measurements on higher $J$ transitions of these excited states than those discussed in this paper is clearly demonstrated. 


\section{Discussion}

The rotational constant $B_{\mathrm{v}}$ is given by

$$
\begin{aligned}
B_{\mathrm{v}}=B_{\mathrm{e}} & -\sum_{i} \alpha_{i}\left(v_{i}+d_{i} / 2\right) \\
& +\sum_{i j} \gamma_{i j}\left(v_{i}+d_{1} / 2\right)\left(v_{j}+d_{j} / 2\right) .
\end{aligned}
$$

Thus for the various overtones of the low frequency degenerate bending mode $\nu_{5}$ we have

$$
B_{\mathrm{v}}=B_{\mathrm{e}}-\alpha_{5}\left(v_{5}+1\right)+\gamma_{5}\left(v_{5}+1\right)^{2} .
$$

Thus from the experimental values of $B_{\mathrm{v}}-B_{0}$ for the states $\left(0,0,1^{1}\right),\left(0,0^{0}, 2^{0,2}\right),\left(0,0^{0}, 3^{1}\right)$ and $\left(0,0^{0}, 4^{0,2}\right)$ we can calculate both $\alpha_{5}$ and $\gamma_{5}$. The values calculated are for HCCF $\alpha_{5}=-28.50 \mathrm{MHz}$, $\gamma_{5}=-0.33 \mathrm{MHz}$ for DCCF $\alpha_{5}=-25.78 \mathrm{MHz}$, $\gamma_{5}=-0.43 \mathrm{MHz}$.

In the determination of these constants it was assumed that no perturbations were present and the results are consequently an average over the observed values of $B_{0}-B_{\mathrm{v}}$. The observations on DCCF are reproduced better by the above constants than are those of HCCF, where the effects of minor perturbations were apparent. The extension of this procedure to states involving $v_{4}$ and combination states involving $v_{4}$ and $\nu_{5}$ was limited by the data available, but the following sets of internally consistent constants were produced:

For HCCF from consideration of the states $\left(0,1^{1}, 0\right),\left(0,2^{0,2}, 0\right),\left(0,(1,1)^{0,2}\right)$ and $\left(0,(1,2)^{1}\right)$ the constants $\alpha_{4}=-9.66 \mathrm{MHz}, \gamma_{4}=-0.28 \mathrm{MHz}$ and $\gamma_{45}=-0.02 \mathrm{MHz}$ were extracted. In the case of DCCF a problem was immediately encountered that from the value of $\left(B_{0}-B_{\mathrm{v}}\right)$ for the state $\left(0,2^{0,2}, 0\right)$ it appears that $\gamma_{4}$ must have a positive sign whereas the combination states indicate a negative sign. Since in all other states a negative

[1] M. Winnewisser and B. P. Winnewisser, J. Molec. Spectr. 41, 143 (1972).

[2] T. Tanaka, C. Yamada, and E. Hirota, J. Molec. Spectr. 63, 142 (1976).

[3] G. R. Hunt and M. K. Wilson, J. Chem. Phys. 34, 1301 (1961).

[4] J. K. Tyler and J. Sheridan, Trans. Faraday Soc. 59, 2661 (1963).

[5] H. Jones, M. Takami, and J. Sheridan, Z. Naturforsch. in press (1979).

[6] H. Jones, J. Sheridan, and O. L. Stiefvater, Z. Naturforsch. 32a, 866 (1977). sign was associated with this constant we considered the $\left(0,2^{0,2}, 0\right)$ state to be perturbed and excluded it from the calculation. From consideration of states $\left(0,1^{1}, 0\right), \quad\left(0,(1,1)^{0,2}\right), \quad\left(0,(2,1)^{1}\right) \quad$ and $\left(0,(1,2)^{1}\right)$ the constants $\alpha_{4}=-15.50 \mathrm{MHz}, \gamma_{4}=$ $-0.14 \mathrm{MHz}$ and $\gamma_{45} \cong 0.0 \mathrm{MHz}$ were calculated.

The constants produced as a result of these simple calculations in which all extraneous effects, such as perturbations, were neglect may prove to have considerable errors attached to them. This is particularly true of the $\gamma$ constants and consequently these constants should be considered with due caution.

\section{Note Added in Proof}

The results from a recent Stark-laser spectroscopy study of the $\nu_{1}$ band of DCCF [13] lead us to believe that the $R(10)$ transition of this band is possibly in coincidence with the $\mathrm{P}(14)$ laser line. However new experiments in which the $J=10 \rightarrow 11$ excited state transition was observed failed to produce observable double resonance signals.

Since this new information does not increase the degree of certainty about our observations we propose to let the description of our initial experiments stand as an aid to future studies of this problem.

\section{Acknowledgements}

The authors wish to thank Dr. Brenda Winnewisser and Dr. H. K. Bodenseh for their advice on the treatment of the excited state spectra and for the use of a program for fitting the direct $l$-type doubling transitions. We also wish to thank Dr. W. Hüttner for helpful discussions. This work is supported by the Deutsche Forschungsgemeinschaft under the title $\mathrm{Ru} 48 / 29$.

[7] W. Hüttner, H. K. Bodenseh, and P. Nowicki, Mol. Phys. in press.

[8] H. G. Viehe and E. Franchimont, Chem. Ber. 95, 319 (1962).

[9] H. Jones, Appl. Phys. 14, 169 (1977).

[10] G. Amat and H. H. Nielsen, J. Molec. Spectr. 2, 152 (1958).

[11] H. H. Nielsen and W. H. Shaffer, J. Chem. Phys. 11, 140 (1943).

[12] S. Reichman, P. C. Johnson, and J. Overend, Spectrochim. Acta 25 A, 245 (1968).

[13] T. Tanaka, private communication. 